\title{
On the Problem of Metal-Insulator Transitions in Vanadium Oxides
}

\author{
A. L. Pergament, G. B. Stefanovich, N. A. Kuldin, and A. A. Velichko \\ Physics and Technology Department, Petrozavodsk State University, Petrozavodsk 185910, Russia
}

Correspondence should be addressed to G. B. Stefanovich; gstef@yandex.ru

Received 14 May 2013; Accepted 16 June 2013

Academic Editors: A. N. Kocharian, A. Krimmel, and A. Oyamada

Copyright (C) 2013 A. L. Pergament et al. This is an open access article distributed under the Creative Commons Attribution License, which permits unrestricted use, distribution, and reproduction in any medium, provided the original work is properly cited.

\begin{abstract}
The problem of metal-insulator transition is considered. It is shown that the Mott criterion $a_{\mathrm{B}}\left(n_{\mathrm{c}}\right)^{1 / 3} \approx 0.25$ is applicable not only to heavily doped semiconductors but also to many other materials, including some transition-metal compounds, such as vanadium oxides (particularly, $\mathrm{VO}_{2}$ and $\mathrm{V}_{2} \mathrm{O}_{3}$ ). The low-temperature transition ("paramagnetic metal-antiferromagnetic insulator") in vanadium sesquioxide is described on the basis of this concept in terms of an intervening phase, between metal and insulator states, with divergent dielectric constant and effective charge carrier mass. Recent communications concerning a possible "metalinsulator transition" in vanadium pentoxide are also discussed.
\end{abstract}

\section{Introduction}

Strongly correlated transition metal oxides exhibiting metalinsulator transitions (MIT) are currently considered as basic functional materials of oxide electronics [1]. That is why the theoretical models describing MITs are of such importance. Vanadium oxide Magneli phases, forming the homologous series $\mathrm{V}_{n} \mathrm{O}_{2 n-1}(3 \leq n \leq 9)$, may be considered as intermediate structures between the end members $\mathrm{VO}_{2}(n \rightarrow$ $\infty)$ and $\mathrm{V}_{2} \mathrm{O}_{3}(n=2)$. With one exception $\left(\mathrm{V}_{7} \mathrm{O}_{13}\right)$, the vanadium Magneli phases exhibit MITs [2] as functions of temperature-see Figure 1 and [1-10].

In recent years, a number of papers [4-6] have appeared concerning a "metal-insulator transition" in $\mathrm{V}_{2} \mathrm{O}_{5}$ [3]. Here, we do not intend to dispute scientific aspects of these works, and the object of our discussion will be different. The fact is that the term "metal-insulator transition", particularly with respect to vanadium oxides (and not only to these-generally to a variety of transition metal oxides and related materials [7]), has long been used in the scientific literature to describe quite a certain class of phenomena and experimental facts. Thus, in this paper we will comment on the aforecited references [4-6], with a more detailed discussion than that in our brief note [3], in view of the problem of MITs in vanadium oxides. Also, the MITs in $\mathrm{V}_{2} \mathrm{O}_{3}$ are discussed, and an attempt is made to describe them in terms of a model based on the Mott criterion concept, developing some ideas of our previous work [7]. However, first, in Section 2.1, we have to acquaint a reader with the main general points of discussion and recall some results obtained earlier for vanadium dioxide and other related materials.

\section{Mott Metal-Insulator Transitions in Vanadium Oxides}

2.1. Strongly Correlated Systems and Mott Transition. At present, there is no unified approach to interpret the electronic properties of transition metal oxides [8]. The band theory and the crystal field theory are the two limiting descriptions of the outer electrons in solids, and the essence of difference between metals and insulators can be understood in terms of these two models. For example, in the elementary band theory, when atoms are combined to form a macroscopic bulk sample with $N$ primitive unit cells, each electron energy level per unit cell gives rise to $N$ electron states in the energy band of a crystal. Whether the material will be a metal or an insulator depends on whether the band is filled partially or completely, or whether or not the two bands will intersect. In the semiconductor state, the number of charge carriers can be increased by adding an impurity, and when the dopant concentration exceeds a certain value $\left(n_{\mathrm{c}}\right)$, a semiconductor undergoes a Mott MIT [9] into a metal state. 


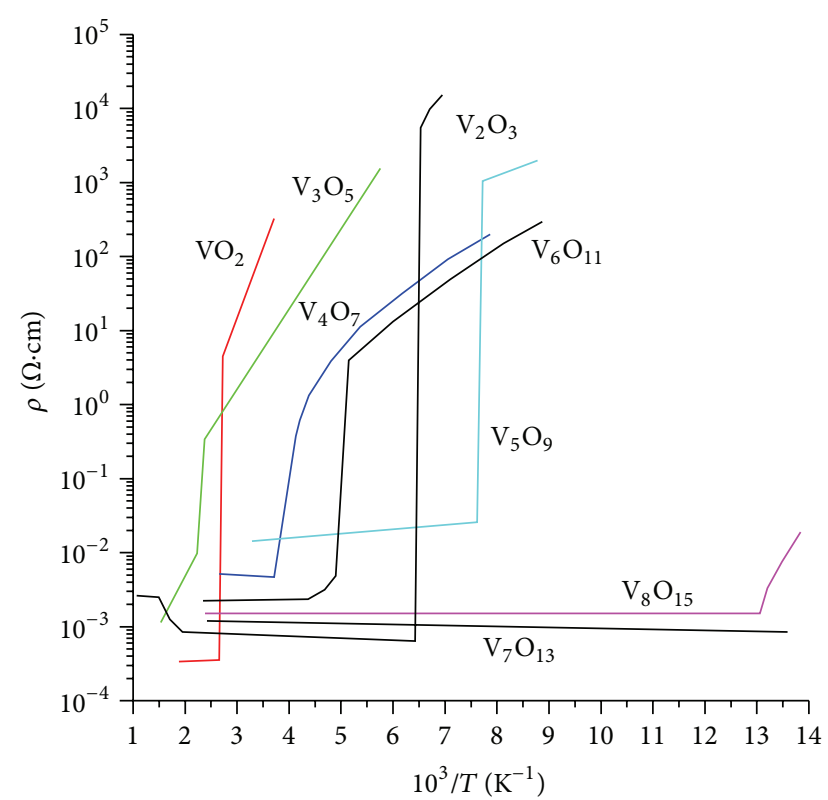

FIGURE 1: Resistivity versus reciprocal temperature for vanadium oxides.

Similar consideration is applicable to other compounds of variable composition, such as, for instance, high-temperature superconductors HTCS [8]. The value of $n_{\mathrm{c}}$ is given by the well-known relation, the Mott criterion [9]

$$
a_{\mathrm{B}} n_{\mathrm{c}}^{1 / 3} \approx 0.25,
$$

where $a_{\mathrm{B}}$ is the effective Bohr radius for the charge carrier localization on an impurity ion.

As was repeatedly noted previously $[7,10]$, the Mott criterion expressed by (1) proved to be very successful in describing MITs in various ordered systems, doped semiconductors [8-11], and transition metal compounds [7] included (see Figure 2 and Table 1). The Hubbard model suggests that "interactions between electrons are accounted for, via the repulsive Hubbard $U$ term, only when they are on the same site" [11]. Then Anderson has shown that, for disordered systems, at a certain degree of disorder, all the electrons would be localized, which renders the system nonconducting [11, 14]. In compounds exhibiting a temperature- or pressure-induced MIT, instead of the one caused by doping (substitution) or disorder, the Mott transition theory is rather more sophisticated [7-11]. As compared with the above simple picture for doped semiconductors, some new important phenomena are observed in compounds of $d$ and $f$ elements. For example, transition metal compounds containing atoms with unfilled $d$-shells form complex systems of phases with multiple oxidation states and mixed valence. These compounds belong to a class of strongly correlated systems, and strong electronelectron correlations are associated with a specific behavior of $d$-electrons. Since the $d$-bands are narrow, the energy of electron-electron Coulomb interactions is of the order of the bandwidth $B$ (and, hence, the electron kinetic energy), that is, $U \sim B$. It is commonly accepted by now that strong correlation effects are responsible for some unique properties of such
TABLE 1: The effective Bohr radius $a_{\mathrm{B}}=\varepsilon \hbar^{2} / m^{*} e^{2}$ and the critical carrier density for an MIT in some compounds (numbers of rows in the table correspond to numbers of points in Figure 2).

\begin{tabular}{lcccc}
\hline No. & Material & $a_{B}, \mathrm{~nm}$ & $n_{c}, 10^{17} \mathrm{~cm}^{-3}$ & References \\
\hline 1 & $\mathrm{Si}: \mathrm{P}$ & 1.35 & 30 & \\
2 & $\mathrm{Si}: \mathrm{B}$ & 1.41 & 41 & \\
3 & $\mathrm{Si}: \mathrm{Bi}$ & 0.88 & 140 & \\
4 & $\mathrm{Ge}: \mathrm{Sb}$ & 4.74 & 1.5 & \\
5 & $\mathrm{n}-\mathrm{GaAs}$ & 9.67 & 0.12 & \\
6 & $\mathrm{n}-\mathrm{InSb}$ & 57.7 & 0.001 & {$[7,10]$} \\
7 & $\mathrm{CdS}: \mathrm{In}$ & 3.5 & 2.5 & \\
8 & $\mathrm{CdS}: \mathrm{Cl}$ & 3.3 & 9.9 & \\
9 & $\mathrm{GaP}: \mathrm{Zn}$ & 1.1 & 130 & \\
10 & $\mathrm{Cu}: \mathrm{Ar}$ & 0.09 & $2 \cdot 10^{5}$ & \\
11 & $\mathrm{YBa}_{2} \mathrm{Cu} \mathrm{O}_{7}$ & 0.45 & 1800 & {$[7,12]$} \\
12 & $\mathrm{NH}_{3}: \mathrm{Na}$ & 0.8 & 340 & {$[7]$} \\
\hline 13 & $\mathrm{VO}_{2}$ & 1.8 & 28 & {$[15-18]$} \\
\hline 14 & $\mathrm{~V}_{2} \mathrm{O}_{3}$ & 1.18 & 100 & \\
\hline 15 & $\mathrm{~V}_{2} \mathrm{O}_{3}(\mathrm{AFI})$ & $\sim 1.2$ & 0.021 & \\
\hline 16 & $\mathrm{SmS}_{10}$ & $0.73^{(*)}$ & $700[15,16]$ & \\
\hline 17 & $\mathrm{Sr}_{1-x} \mathrm{La}_{x} \mathrm{TiO}$ & 0.97 & 30 & \\
\hline$(*)$ & $\mathrm{For}_{3} m^{*}=1.3 m_{e}[17]$ and $\varepsilon=18[18]$. & & \\
\hline
\end{tabular}

materials as HTCS cuprates, materials with metal-insulator transitions, heavy-fermion superconductors, ferromagnetic perovskites with colossal magnetoresistance, and so forth.

Another well-known example of the influence of correlation effects on the band structure is a "correlated insulator" (NiO, e.g.). Insulators of this type are also known as Mott insulators, for it was Mott's suggestion that electron repulsion is responsible for a breakdown of normal band properties for the $d$-electrons, which was later formalized in the Hubbard model $[9,11]$. Note that high- and low-temperature phases of vanadium sesquioxide doped by chromium to $c a 1$ at.\% (Figure 3) are just the ones representing, amongst others, such Mott insulator phases [9].

As far as vanadium oxides concerns, it has been shown [7] that, in the case of vanadium dioxide, as well as for a high-temperature transition "paramagnetic insulator-paramagnetic metal" in vanadium sesquioxide, the relation (1) is completely fulfilled.

In particular, for vanadium dioxide, the value of the critical density $n_{c}$ is of the order of $n_{s}$-the equilibrium electron density in the conduction band of $\mathrm{VO}_{2}$ in the lowtemperature semiconducting phase at $T \rightarrow T_{t}$ (where $T_{t}$ is the transition temperature, $\sim 340 \mathrm{~K}$ for vanadium dioxidesee Figure 1). Next, $a_{B}=\hbar^{2} \varepsilon / e^{2} m^{*}$, where $\varepsilon$ and $m^{*}$ are the dielectric constant and effective mass, respectively. For $\varepsilon=100$ and $m^{*}=3 m_{e}[7,12]$, this yields $a_{\mathrm{B}}=1.77 \mathrm{~nm}$ in vanadium dioxide, and the product of $a_{\mathrm{B}}$ and $\left(n_{\mathrm{c}}\right)^{1 / 3}$ turns out to be just that as predicted by (1) - point 13 in Figure 2 .

2.2. Vanadium Sesquioxide. It is really noteworthy that the Mott criterion (1) works in the case of vanadium dioxide and a PI-PM transition in vanadium sesquioxide (see rows 13 and 14 in Table 1, as well as corresponding points in Figure 2) and not 


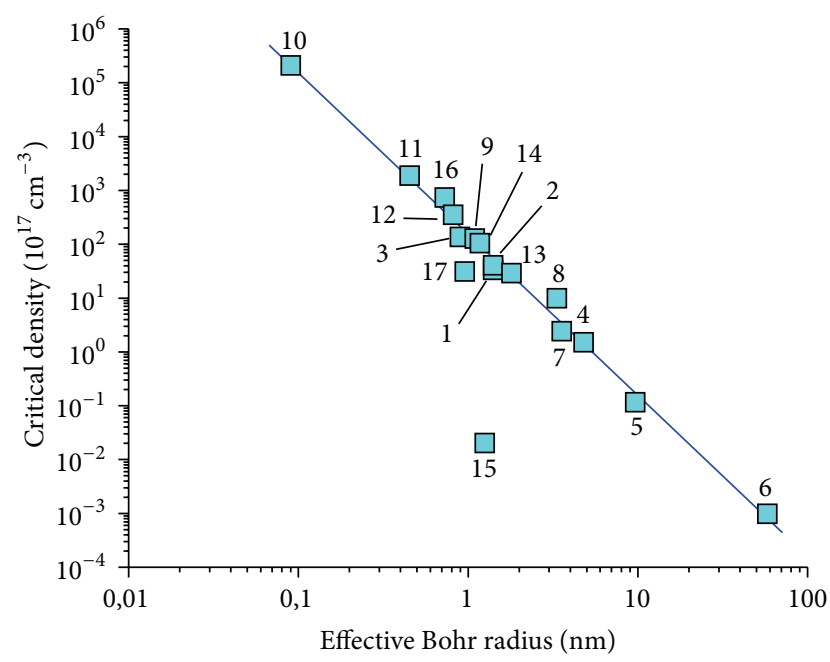

Figure 2: Correlation between the effective Bohr radius and the critical densities for different systems (see Table 1). Only some representative materials are included; other examples one can find in $[7,10]$. Straight line corresponds to (1).

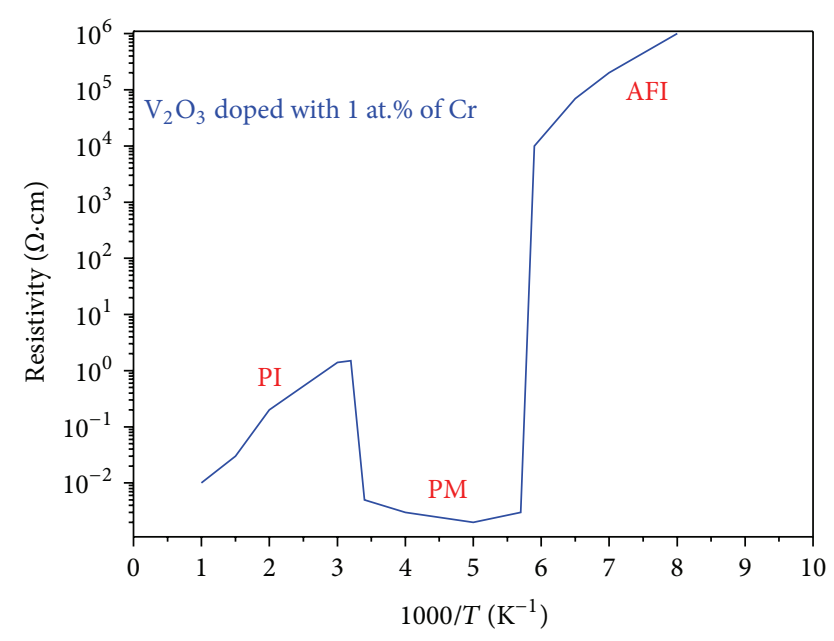

Figure 3: Temperature dependence of resistivity for $\left(\mathrm{V}_{1-x} \mathrm{Cr}_{x}\right)_{2} \mathrm{O}_{3}$ : Cr with $x=0.012$ [8-11]. AFI-antiferromagnetic insulator, PM-paramagnetic metal, and PI-paramagnetic insulator.

only for doped semiconductors or some other well-known Mott insulators, like $\mathrm{YBa}_{2} \mathrm{Cu}_{3} \mathrm{O}_{7-x}$ or $\mathrm{Sr}_{1-x} \mathrm{La}_{x} \mathrm{TiO}_{3}$.

However, in the case of a low-temperature AFI $\rightarrow$ PM transition in $\mathrm{V}_{2} \mathrm{O}_{3}$, the situation becomes more complicated. For this transition, as was shown in [7], the product of $a_{\mathrm{B}}$ and $\left(n_{\mathrm{c}}\right)^{1 / 3}$ is far less than 0.25 (Figure 2, point 15); that is, the Debye screening length $L_{\mathrm{D} \text {,quant }}$ is far in excess of $a_{\mathrm{B}}$, which suggests that the Mott transition to a metallic state would seem hardly probable. On the other hand, in a classic case, the screening length can be written as

$$
L_{\text {D,class }}=\sqrt{\frac{k_{\mathrm{B}} T \varepsilon_{0}}{4 \pi e^{2} n}},
$$

where $k_{\mathrm{B}}$ and $\varepsilon_{0}$ are the Boltzmann and vacuum permittivity constants and $n$ corresponds to the value of $n_{\mathrm{c}}$ for the transition point. In this case one still obtains $L_{\mathrm{D}}>a_{\mathrm{B}}$, because, for $T=T_{t}=170 \mathrm{~K}$ (see Figure 3), (2) yields $L_{\mathrm{D}}=$ $5.6 \mathrm{~nm}$, while $a_{\mathrm{B}}$ is equal to only $1.2 \mathrm{~nm}$ (this quantity can be obtained for $\mathrm{V}_{2} \mathrm{O}_{3}$ from calculation similar to that carried out for $\mathrm{VO}_{2}$ above but with $m^{*}=9 m_{e}$ and $\varepsilon=200$ [7]).

Generally, expressions of the type $L_{\mathrm{D}}=R$ (where $R$ is the electron localization radius, for instance, $a_{\mathrm{B}}$ ) or $B=U$ (where $B$ and $U$ are the kinetic and potential electron energies, resp.) are nearly equivalent to the Mott criterion given by (1): see, for example, discussions in the works $[12,20]$ related to this issue. Note that the value of $a_{\mathrm{B}}$ is determined by the dielectric constant and effective mass. Unfortunately, we do not know the exact values of $\varepsilon$ and $m^{*}$ near the phase transition because of their divergence (or unrestricted growth), and probably that very factor is responsible for the fulfillment of the equality $L_{\mathrm{D}}=R$. This situation is illustrated in Figure 4 which suggests that an increase of $R=a_{\mathrm{B}}$ due to increased $\varepsilon$ close to the MIT, as well as a decrease of $L_{\mathrm{D}}$ due to increased $n$, would result in the intersection of the two curves at the transition point. These speculations show that the low-temperature AFI $\rightarrow$ PM transition in $\mathrm{V}_{2} \mathrm{O}_{3}$ might also be described from the position of the Mott MIT. In other words, the dependence $a_{\mathrm{B}}(T)$ in Figure 4 can account for the fulfillment of relation (1) in the case of the transition from AFI to PM in chromiumdoped vanadium sesquioxide and solve thereby the problem with this transition formulated in the work [7].

Thus, we come to a conclusion that the MITs in some lower vanadium oxides, namely, $\mathrm{VO}_{2}$ and $\mathrm{V}_{2} \mathrm{O}_{3}$, might be fairly satisfactory described in terms of the Mott transition model. It is to be noted that the relations between structural and electronic properties should be taken into account when describing an exact MIT mechanism both in these oxides and in other vanadium Magneli phases [21]. Structural aspects are of particular importance, for instance, in $\mathrm{VO}_{2}$ where the MIT is often described in terms of a "Mott-Peierls" (or correlation-assisted Peierls) transition [22, 23], as well as in $\mathrm{V}_{7} \mathrm{O}_{13}$ where neither of the two mechanisms (i.e., electron correlation effects or Peierls-like structural instabilities) is strong enough to induce a transition [21].

2.3. Vanadium Pentoxide: Is There a Metal-Insulator Transition in This Highest-Valence Vanadium Oxide? Now, returning to the problem of a possible MIT in the highest vanadium oxide, $\mathrm{V}_{2} \mathrm{O}_{5}$, we should say that the above-described picture exploded when we learned [3] about a "metal-insulator transition in vanadium pentoxide" [4-6]. This oxide is not a member of the Magneli $\mathrm{V}_{n} \mathrm{O}_{2 n-1}$ series, and it has ever been known as a semiconductor with the band gap width of $\sim 2.2 \mathrm{eV}$ [8] exhibiting no trend to a transition into metallic state. Here the point is that we are dealing with a change in phase composition, which is not the same thing. We are presented with a phase transition of vanadium pentoxide upon heating up to $\sim 280^{\circ} \mathrm{C}$ [5] into some other vanadium oxide phases, exhibiting metallic properties at these temperatures, as purportedly an MIT in $\mathrm{V}_{2} \mathrm{O}_{5}$. Actually, the transformation described in the article [5] is neither a temperature-induced MIT, nor is it even a composition-induced MIT as, for 


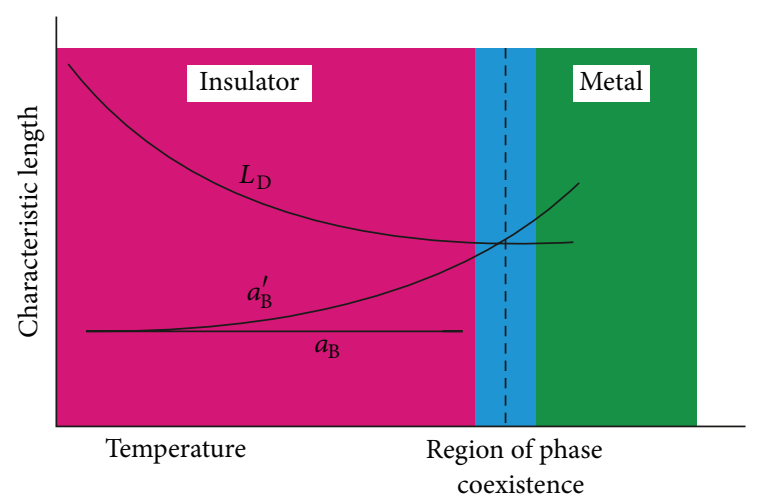

FIGURE 4: Schematic temperature dependences of the Debye screening length and localization radius. If account is taken of the dependence of $a_{\mathrm{B}}$ on $T$ via the divergence of $\varepsilon$ near the transition point (curve $a_{\mathrm{B}}^{\prime}$ ), the transition does occur (vertical dashed line). For more detailed discussion of the $L_{\mathrm{D}}$ dependence on $T$ see [7]. A region of phase coexistence (termed as an "intermediate state" [12] or a "strongly correlated metal" [13] in case of $\mathrm{VO}_{2}$ ) with a divergent effective mass is also shown.

example, a transition " $\mathrm{V}_{2} \mathrm{O}_{5}$-nonstoichiometric $\mathrm{V}_{2} \mathrm{O}_{5-\delta}$." This transformation is associated with a reduction of $\mathrm{V}_{2} \mathrm{O}_{5}$, we repeat once more, to lower oxides: "when $\mathrm{V}_{2} \mathrm{O}_{5}$ is subjected to high temperature, the material loses oxygen. This induces a structural inhomogeneity, resulting in a mixing of phases such as $\mathrm{V}_{2} \mathrm{O}_{5}, \mathrm{~V}_{6} \mathrm{O}_{13}, \mathrm{~V}_{2} \mathrm{O}_{3}$ " [5].

Such a misunderstanding seems to originate from the work [24] where experiments on memory switching in thinfilm $\mathrm{V}_{2} \mathrm{O}_{5}$-based structures have been described. To interpret the results of these experiments, the authors of [24] put forward a hypothesis of some "glass-to-metal" transition in amorphous vanadium pentoxide. Afterwards, in the literature, this assumption somehow transformed into an idea of an MIT in $\mathrm{V}_{2} \mathrm{O}_{5}$ which was further widely circulated (with no efforts to delve into the problem) and much referred to. The reference was first made in [25] (just as with an MIT in $\mathrm{V}_{2} \mathrm{O}_{5}$ at $T_{t}=257^{\circ} \mathrm{C}$ ), followed by a few refutations $[26,27]$. The subject could have seemed exhausted. Meanwhile, in the papers [28-33], we have again encountered the same statement (and again with references to either [24] or some other old works) and, finally, it has been reported on an "MIT in $\mathrm{V}_{2} \mathrm{O}_{5}$," as it is asserted in the titles of the articles $[4,5]$.

One cannot but admit that everything is correct in those articles (as to the results presented), but entering such terms in the article titles seems to be not quite appropriate. There is certainly no metal-insulator transition in vanadium pentoxide in the commonly accepted sense discussed above. If one even would try to put a corresponding point in Figure 2, it will just exceed the bounds of the figure, well below the abscissa axis, solely because of too low carrier density in dielectric $\mathrm{V}_{2} \mathrm{O}_{5}$.

\section{Conclusion}

In conclusion, it is shown in the present study that the Mott criterion is applicable not only to heavily doped semiconductors but also to many other compounds, including such materials, important for the MIT problem on the whole, as vanadium oxides, particularly, $\mathrm{VO}_{2}$ and $\mathrm{V}_{2} \mathrm{O}_{3}$. In order to verify model approximations introduced in this article, additional experimental data are likely to be needed. Obviously, further studies seem to be of importance, focused on thorough measurements of the dielectric constant, effective mass, and carrier mobility values of both vanadium oxide Magneli phases [21, 34-37] and other strongly correlated $f$ - and $d$ compounds undergoing Mott metal-insulator transitions.

The problem of effective mass is of especial importance. We recall that the value of $m^{*}$ is necessary in order to calculate the effective Bohr radius $a_{\mathrm{B}}$ in the Mott criterion (1). Meanwhile, the accurate charge carrier effective mass values are not always known in case of strongly correlated transition metal compounds. Even for the much-studied vanadium dioxide, the experimental values obtained by various authors differ nearly by an order of magnitude [36]. The difficulties and vagueness while determining an effective mass are well known; these are due to both the quality of a sample and the measuring technique. Optical methods (from measurements of the plasma frequency) and electrical (transport) measurements yield as a rule two different magnitudes, which is no wonder, since the values of $m^{*}$ of DOS (density of states) and the band effective mass should not necessarily coincide [13]. Furthermore, in anisotropic substances, this physical quantity depends on direction. An approach to calculate the effective masses in vanadium oxide Magneli phases has been developed in [36]; however, more efforts, both theoretical and experimental, are needed in this direction.

As to the MIT in vanadium pentoxide [4-6, 25, 28-33], it should be emphasized once again that the scientific importance and correctness of results of the works $[4,5]$ are certainly beyond question, just we would suggest do not use the term "metal-insulator transition", for this is confusing in such a context, as we have tried to show in Section 2.3. The aforesaid, however, does not abolish a feasibility of a temperatureinduced MIT in nonstoichiometric [38] or doped [6] $\mathrm{V}_{2} \mathrm{O}_{5}$. For example, in vanadium dioxide, doping with tungsten to ca. 10-15 at.\% leads to metallization without the material heating up to $T=T_{t}$ [39]. However, this situation is not the same as that described in the work [4] for vanadium pentoxide, because the material $\left(\mathrm{VO}_{2}\right)$ does not transform into another vanadium oxide Magneli phase under such an alloying.

Finally, we would like to reiterate the idea that we have started this paper with; that is, the problems discussed here are not of solely abstract scientific interest, but they are of importance from the practical viewpoint too, since the materials exhibiting MIT find an application for a lot of various electronic and optical devices. It is appropriate here to quote an excerpt from the review [1], where the authors "discuss the exploration of correlated oxide phase transitions in novel electronics, photonics, and related devices. Although this area has intrigued researchers for several decades ever since the original observation of MIT in oxides, the twentyfirst-century grand challenge of continued innovation in information processing sciences beyond CMOS scaling creates an opportunity to seriously consider alternate computing vectors that may offer unique advantages". Particularly, vanadium dioxide can be used as a material for thermochromic 
indicators, optical filters and controllers, bolometers, thinfilm transistors and switches, and many other optical and electronic devices.

Note that vanadium pentoxide has also drawn considerable interest in the past decades due to multiple potential applications such as electrochromic devices, optical switching devices, reversible cathode materials for Li batteries, and gas sensors [32]. Also, $\mathrm{V}_{2} \mathrm{O}_{5}$ exhibits thermochromic properties, likewise $\mathrm{VO}_{2}$. It should be stressed however that the thermochromic and photochromic effects in vanadium pentoxide are both due to a reversible hydrogen injection-extraction [40], and, hence, thermochromism in $\mathrm{V}_{2} \mathrm{O}_{5}$ is not associated with an MIT as it is mistakenly stated, for example, in the work [29]. Recently, vanadium pentoxide was synthesized in the form of nanowires, nanorods, and nanofibers $[6,32]$. The potential use of such $\mathrm{V}_{2} \mathrm{O}_{5}$ nanoobjects provides excellent possibilities for designing new electronic and optoelectronic nanodevices. That is why the question about an MIT in $\mathrm{V}_{2} \mathrm{O}_{5}$ is of especial scientific interest and practical importance.

\section{Acknowledgments}

This work was supported by the Ministry of Education and Science of Russian Federation "Scientific and Educational Community of Innovation Russia (2009-2013)" Program through Contracts no. 14.740.11.0895, no. 14.740.11.0137, and no. 16.740.11.0562 and "Development of Scientific Potential of High School" Program, Grants no. 2.3282.2011 and no. 2.2774.2011, as well as the Strategic Development Program of Petrozavodsk State University (2012-2016). The authors also thank V. N. Andreev for useful discussions and V. P. Novikova for her help with the paper preparation.

\section{References}

[1] Z. Yang, C. Ko, and S. Ramanathan, "Oxide electronics utilizing ultrafast metal-insulator transitions," Annual Review of Materials Research, vol. 41, pp. 337-367, 2011.

[2] F. J. Morin, "Oxides which show a metal-to-insulator transition at the neel temperature," Physical Review Letters, vol. 3, no. 1, pp. 34-36, 1959.

[3] A. Pergament, G. Stefanovich, and V. Andreev, "Comment on "Metal-insulator transition without structural phase transition in $\mathrm{V}_{2} \mathrm{O}_{5}$ film" [Appl. Phys. Lett. 98, 131907 (2011)]," Applied Physics Letters, vol. 102, Article ID 176101, 1 page, 2013.

[4] R.-P. Blum, H. Niehus, C. Hucho et al., "Surface metal-insulator transition on a vanadium pentoxide (001) single crystal," Physical Review Letters, vol. 99, no. 22, Article ID 226103, 2007.

[5] M. Kang, I. Kim, S. W. Kim, J.-W. Ryu, and H. Y. Park, "Metalinsulator transition without structural phase transition in $\mathrm{V}_{2} \mathrm{O}_{5}$ film," Applied Physics Letters, vol. 98, no. 13, Article ID 131907, 2011.

[6] T. Wu, C. J. Partridge, S. Banerjiee, and G. Sambandamurthy, "Metal-insulator transition in individual nanowires of doped $\mathrm{V}_{2} \mathrm{O}_{5}$," American Physical Society, APS March Meeting, abstract no. V16.007, 2010.

[7] A. Pergament and G. Stefanovich, "Insulator-to-metal transition in vanadium sesquioxide: does the Mott criterion work in this case?" Phase Transitions, vol. 85, no. 3, pp. 185-194, 2012.
[8] P. A. Cox, Transition Metal Oxides: An Introduction to Their Electronic Structure and Properties, Clarendon Press, Oxford, UK, 1992.

[9] N. F. Mott, Metal-Insulator Transitions, Taylor and Francis, London, UK, 1990.

[10] P. P. Edwards, T. V. Ramakrishnan, and C. N. R. Rao, “The metalnonmetal transition: a global perspective," Journal of Physical Chemistry, vol. 99, no. 15, pp. 5228-5239, 1995.

[11] R. Redmer, F. Hensel, and B. Holst, Eds., Metal-To-Nonmetal Transitions, vol. 132 of Springer Series in Materials Science, Springer, New York, NY, USA, 2010.

[12] A. Pergament and A. Morak, "Photoinduced metal-insulator transitions: critical concentration and coherence length," Journal of Physics A, vol. 39, no. 17, pp. 4619-4623, 2006.

[13] M. M. Qazilbash, M. Brehm, B.-G. Chae et al., "Mott transition in $\mathrm{VO}_{2}$ revealed by infrared spectroscopy and nano-imaging," Science, vol. 318, no. 5857, pp. 1750-1753, 2007.

[14] D. Belitz and T. R. Kirkpatrick, “The Anderson-Mott transition," Reviews of Modern Physics, vol. 66, no. 2, pp. 261-380, 1994.

[15] V. V. Kaminskii, A. V. Golubkov, and L. N. Vasilev, "Defect samarium ions and electromotive-force generation in SmS," Physics of the Solid State, vol. 44, no. 8, pp. 1574-1578, 2002.

[16] V. V. Kaminskii, L. N. Vasilev, M. V. Romanova, and S. M. Solov'ev, "The mechanism of the appearance of an electromotive force on heating of SmS single crystals," Physics of the Solid State, vol. 43, pp. 1030-1032, 2001.

[17] B. Batlogg, A. Schlegel, and P. Wachter, "Degree of valence mixing in the metallic phase of SmS and TmSe," Journal de Physique, vol. 37, pp. 267-270, 1976.

[18] V. Železný, J. Petzelt, V. V. Kaminski, M. V. Romanova, and A. V. Golubkov, "Far infrared conductivity and dielectric response of semiconducting SmS," Solid State Communications, vol. 72, no. 1, pp. 43-47, 1989.

[19] S. Hui, Evaluation of Yttrium-doped $\mathrm{SrTiO}_{3}$ as a solid oxide fuel cell anode [Ph.D. thesis], 2000, http://digitalcommons.mcmaster .ca/opendissertations/1659.

[20] J. Spałek, J. Kurzyk, R. Podsiadły, and W. Wójcik, "Extended Hubbard model with the renormalized Wannier wave functions in the correlated state II: quantum critical scaling of the wave function near the Mott-Hubbard transition," European Physical Journal B, vol. 74, no. 1, pp. 63-74, 2010.

[21] U. Schwingenschlögl and V. Eyert, "The vanadium Magnéli phases $\mathrm{V}_{\mathrm{n}} \mathrm{O}_{2 \mathrm{n}-1}$," Annalen der Physik, vol. 13, no. 9, pp. 475-510, 2004.

[22] J. Laverock, A. R. H. Preston, D. Newby Jr. et al., "Photoemission evidence for crossover from Peierls-like to Mott-like transition in highly strained $\mathrm{VO}_{2}$," Physical Review B, vol. 86, no. 19, Article ID 195124, 5 pages, 2012.

[23] S. Kim, K. Kim, C. J. Kang, and B. I. Min, "Correlation-assisted phonon softening and the orbital-selective Peierls transition in $\mathrm{VO}_{2}$," Physical Review B, vol. 87, Article ID 195106, 2013.

[24] G. S. Nadkarni and V. S. Shirodkar, "Experiment and theory for switching in $\mathrm{Al} / \mathrm{V}_{2} \mathrm{O}_{5} / \mathrm{Al}$ devices," Thin Solid Films, vol. 105, no. 2, pp. 115-129, 1983.

[25] E. E. Chain, "Optical properties of vanadium dioxide and vanadium pentoxide thin films," Applied Optics, vol. 30, pp. 27822787, 1991.

[26] H. Jerominek, F. Picard, and D. Vincent, "Vanadium oxide films for optical switching and detection," Optical Engineering, vol. 32, no. 9, pp. 2092-2099, 1993. 
[27] Y. Dachuan, X. Niankan, Z. Jingyu, and Z. Xiulin, "Vanadium dioxide films with good electrical switching property," Journal of Physics D, vol. 29, no. 4, pp. 1051-1057, 1996.

[28] J. Nag, The solid-solid phase transition in vanadium dioxide thin films: synthesis, physics and application [Ph.D. thesis], 2011, http://etd.library.vanderbilt.edu/available/etd-04202011$182358 /$.

[29] P. Kiri, G. Hyett, and R. Binions, "Solid state thermochromic materials," Advanced Materials Letters, vol. 1, no. 2, pp. 86-105, 2010.

[30] J. Nag and R. F. Haglund Jr., "Synthesis of vanadium dioxide thin films and nanoparticles," Journal of Physics Condensed Matter, vol. 20, no. 26, Article ID 264016, 2008.

[31] M. I. Kang, I. K. Kim, E. J. Oh, S. W. Kim, J. W. Ryu, and H. Y. Park, "Dependence of optical properties of vanadium oxide films on crystallization and temperature," Thin Solid Films, vol. 520, no. 6, pp. 2368-2371, 2012.

[32] S. Beke, "A review of the growth of $\mathrm{V}_{2} \mathrm{O}_{5}$ films from 1885 to 2010," Thin Solid Films, vol. 519, no. 6, pp. 1761-1771, 2011.

[33] R. M. Oksuzoglu, P. Bilgic, M. Yildirim, and O. Deniz, "Influence of post-annealing on electrical, structural and optical properties of vanadium oxide thin films," Optics \& Laser Technology, vol. 48, pp. 102-109, 2013.

[34] T. Reeswinkel, D. Music, and J. M. Schneider, "Coulombpotential-dependent decohesion of Magnéli phases," Journal of Physics Condensed Matter, vol. 22, no. 29, Article ID 292203, 2010.

[35] B. Stegemann, M. Klemm, S. Horn, and M. Woydt, "Switching adhesion forces by crossing the metal-insulator transition in Magnéli-type vanadium oxide crystals," Beilstein Journal of Nanotechnology, vol. 2, no. 1, pp. 59-65, 2011.

[36] A. Pergament, "Metal-insulator transition temperatures and excitonic phases in vanadium oxides," ISRN Condensed Matter Physics, vol. 2011, Article ID 60591, 5 pages, 2011.

[37] B. G. Idlis and Y. V. Kopaev, "On the theory of phase transitions in vanadium oxides $\mathrm{V}_{\mathrm{n}} \mathrm{O}_{2 \mathrm{n}-1}$ (Magneli phases)," Solid State Communications, vol. 45, no. 3, pp. 301-304, 1983.

[38] A. L. Pergament, G. B. Stefanovich, V. N. Andreev, and P. A. Boldin, "Electronic instabilities in crystals of transition metal compounds," in Proceedings of the Petrozavodsk State University, no. 6 (127), pp. 87-98, 2012.

[39] A. Pergament, G. Stefanovich, O. Berezina, and D. Kirienko, "Electrical conductivity of tungsten doped vanadium dioxide obtained by the sol-gel technique," Thin Solid Films, vol. 531, pp. 572-576, 2013.

[40] A. I. Gavrilyuk, N. M. Reinov, and F. A. Chudnovskii, "Photochromism and thermochromism in amorphous $\mathrm{V}_{2} \mathrm{O}_{5}$ films," Soviet Technical Physics Letters, vol. 5, pp. 514-515, 1979. 

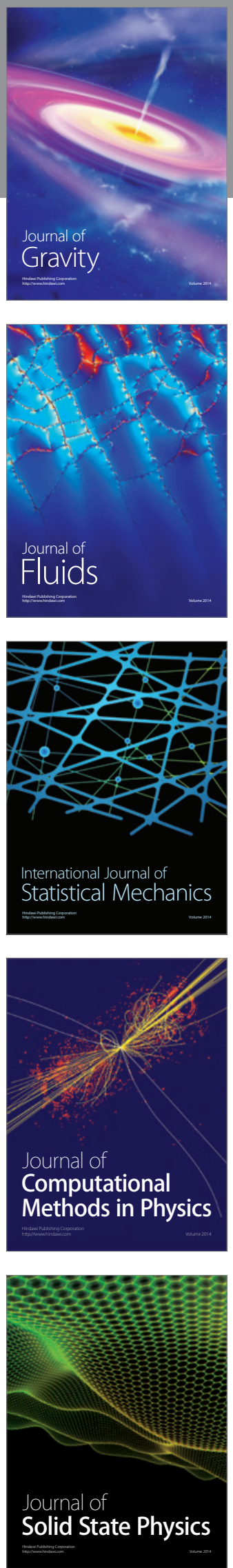

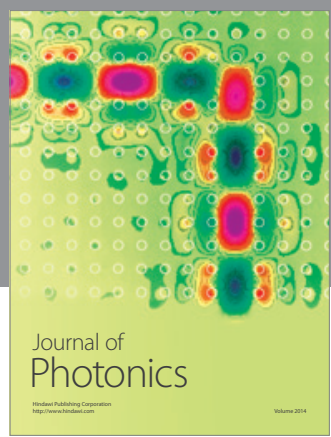

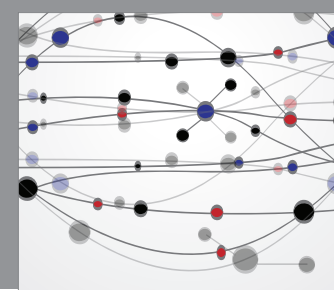

The Scientific World Journal

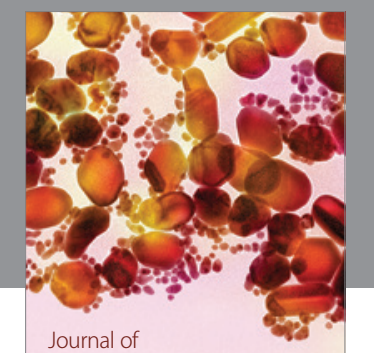

Soft Matter
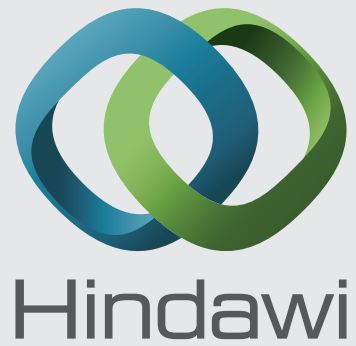

Submit your manuscripts at

http://www.hindawi.com
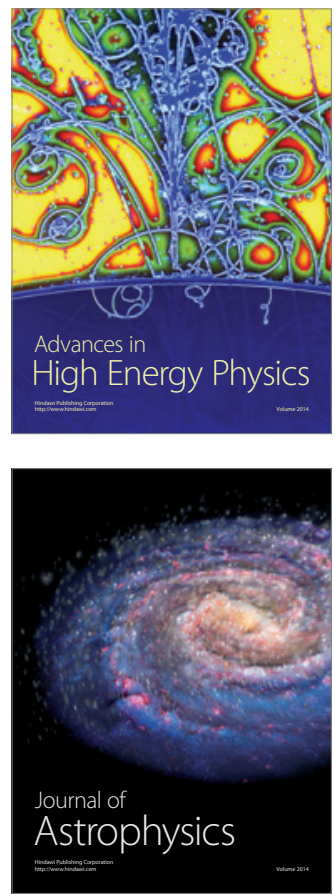
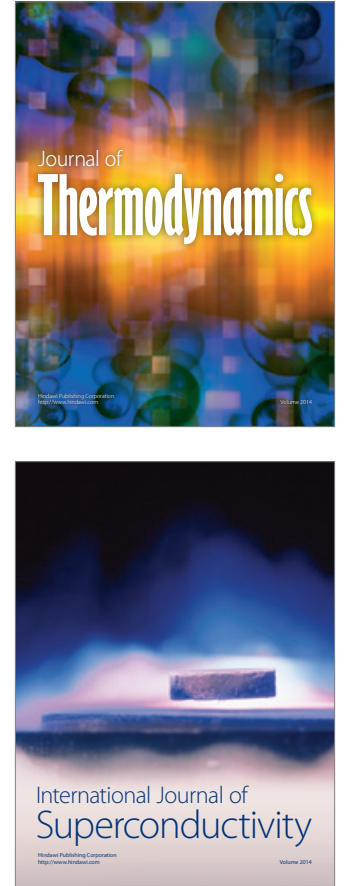
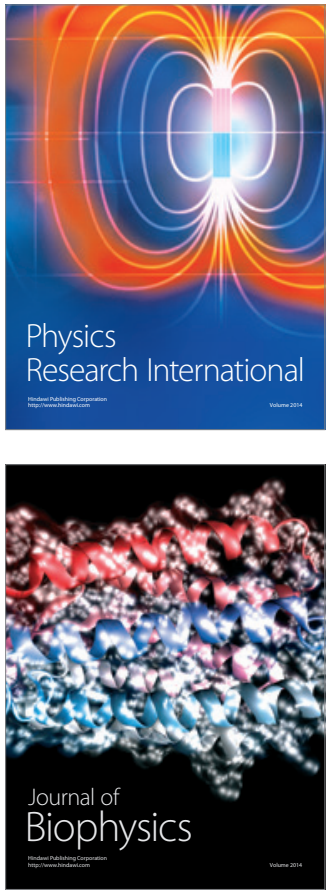
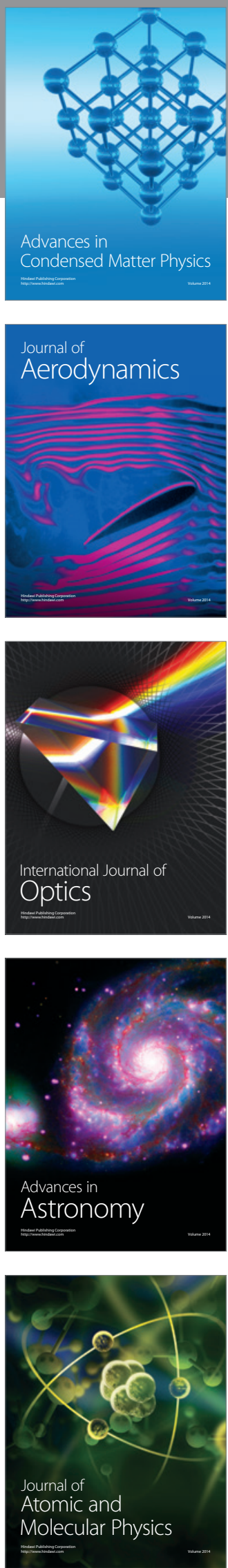